JOURNAL OF THEORETICAL

AND APPLIED MECHANICS

55, 2, pp. 407-420, Warsaw 2017

DOI: $10.15632 /$ jtam-pl.55.2.407

\title{
PARAMETER ESTIMATION OF A DISCRETE MODEL OF A REINFORCED CONCRETE SLAB
}

\author{
Malgorzata Abramowicz, Stefan Berczyński, Tomasz Wróblewski \\ West Pomeranian University of Technology, Szczecin, Poland \\ e-mail:mabramowicz@zut.edu.pl
}

\begin{abstract}
This paper presents parameter estimation of a mathematical model regarding natural vibrations of a reinforced concrete slab. Parameter estimation is based on experiments conducted on a real reinforced concrete slab. Estimated parameters include: substitute longitudinal modulus of elasticity of the reinforced concrete slab, which takes into account longitudinal reinforcement, effective thickness of the reinforced concrete slab and coefficient of damping. Using appropriate criteria during, the process of parameter estimation of the reinforcement concrete slab models has a great impact on obtaining precise results. The estimation criteria are selected in order to achieve consistency of natural vibration frequencies along with the Frequency Response Function measured during experiments with those calculated with the mathematical model. The model and all the calculations have been made using MATLAB programming environment.
\end{abstract}

Keywords: rigid finite element (RFE) model, reinforced concrete slab, estimation criteria, modal parameters, vibrations

\section{Introduction}

Steel-concrete composite beams are the main focus of our previous papers (Berczyński and Gutowski, 2006; Wróblewski et al., 2013). A composite beam is a connection of two or more structural elements made of materials with various properties. An example of such a structure is a steel-concrete composite beam which consists of a steel I-beam and a concrete slab which rests on it. The present paper focuses on the reinforced concrete slab element.

The main topic of the paper is the modelling of vibration of a concrete slab. A 3D RFE (Rigid Finite Element Method) model is presented. Originally developed algorithms of parameter estimation of the reinforced concrete slab model are presented. The estimations were based on experimental results, including beam natural vibration frequencies and FRF (Frequency Response Function) determined empirically (Berczyński and Gutowski, 2006).

As there is no commercially available rigid finite element-based software, an original computer program has been developed in MATLAB environment. The created program can be used to solve the problem of free vibration and to control parameters which can be introduced to describe selected structural elements.

The estimation process is performed in two parallel stages. In the first stage experimental tests are conducted. In the second stage, the computational model of the concrete slab is defined. Solving the eigenproblem gives natural frequencies and FRF runs. Then, using optimisation methods for frequency criteria and FRF runs consistency, parameters of the concrete slab model are chosen to obtain models whose dynamic properties are close to those observed during the experiment. 


\section{Experimental research of vibrations of a reinforced concrete slab}

The experimental stand consisted of two steel bearers at the beam axle spacing of $2 \mathrm{~m}$. The bearers were braced with angle sections. During dynamic tests the beam was suspended on frames with four steel wire ropes $3 \mathrm{~mm}$ in diameter and, in this way, a free beam scheme was implemented. Bearer deformability and its effect of obtained results were considered to be negligible in the scheme. Rope deformability was selected so that frequency vibration typical for solid body in motion was beyond the range of the investigated slab free vibration. A diagram of the test stand as well as the suspended beam are presented in Fig. 1.

(a)



(b)

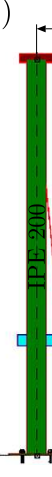

2000



(c)

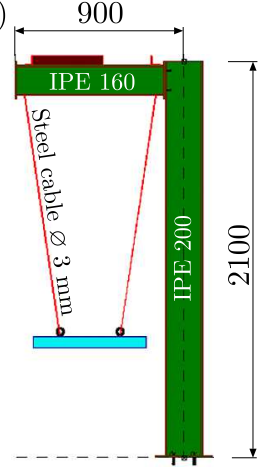

Fig. 1. Test stand: (a) overview, (b) view from the side, (c) head-on view

The aim of the conducted tests was to determine fundamental dynamic characteristics. An impulse excitation was used. Vibration acceleration was a measured value which was considered as the response of the system (Ewins, 2000; Wilde, 2008). Acceleration was measured using triaxial piezoelectric sensors. The sensors were attached with wax to circular steel washers, $25 \mathrm{~mm}$ in diameter, placed on the reinforced concrete slab. The washers were fixed with a modified epoxy resin.

The impulse excitation was performed using a modal hammer KISLER 9726A20000 (500 g). LMS SCADAS III analyser connected to the work station fitted with a computer aided system. Test.Lab package manufactured by LMS was used to record signals. Impact Testing module of the Test.Lab package was used for impulse tests. During each cycle of measurements, acceleration in nine measurement points was recorded. Ten excitation cycles were performed in a predefined spot of the beam. Signal averaging was conducted automatically according to the algorithm implemented in Impact Testing module.

The obtained characteristics of frequency response functions were determined as the ratio of vibration acceleration to the force. The frequency response functions were used to determine the so-called modal model using Modal Analysis module of the Test.Lab system (He and Fu, 2001). A stability analysis method using PolyMAX algorithms was used for parameter estimation of the modal model. This method is based on the frequency response functions.

\section{The analysed reinforced concrete slab}

The reinforced concrete slab (dimensions: $60 \mathrm{~mm}$ thick, $600 \mathrm{~mm}$ wide and $2200 \mathrm{~mm}$ long) was made of $\mathrm{C} 25 / 30$ concrete. The concrete mix was purchased from a local concrete producer. It was made of cement-based class 42.5 with addition of a BV plasticiser. The ratio was $W / C$ of 0.64 with the consistency of S3. The maximum size of aggregate was reduced to $8 \mathrm{~mm}$ owing to a relatively small size of the investigated elements. 
Ribbed steel bars, $6 \mathrm{~mm}$ in diameter made from A-I steel, were used as concrete reinforcement. The longitudinal reinforcement was placed every $75 \mathrm{~mm}$ while transverse reinforcement every $150 \mathrm{~mm}$. A reinforcing fabric from top and bottom was used.

The impulse excitation was applied to the slab at three points (see Fig. 2a): $2-Z$ - vertical impact at the slab axis performed with the modal hammer, $1-Z$ - vertical impact at the edge of slab performed with the modal hammer, $2+Z$ - horizontal impact at the face of slab performed with the modal hammer.

Excitations points in Fig. 2 a are designated with the symbol $\bullet$. Various excitation points aimed at producing different vibration forms of the slab are presented in Table 1.

Table 1. Excitation points and directions

\begin{tabular}{|l|c|c|c|}
\hline \multirow{2}{*}{ Symbol } & \multicolumn{2}{|c|}{ Excitation } & \multirow{2}{*}{ Excited vibration forms } \\
\cline { 2 - 3 } & Point & Direction & \\
\hline \hline $1-Z$ & 1 & $Z$ & flexural and torsional \\
\hline $2-Z$ & 2 & $Z$ & flexural \\
\hline $2+X$ & 2 & $X$ & axial \\
\hline
\end{tabular}

Measurement points were defined at the upper area of the slab, spaced in three rows (9 points in each row) which gave a total of 27 measurement points (see Fig. 2b).

(a)



(b)

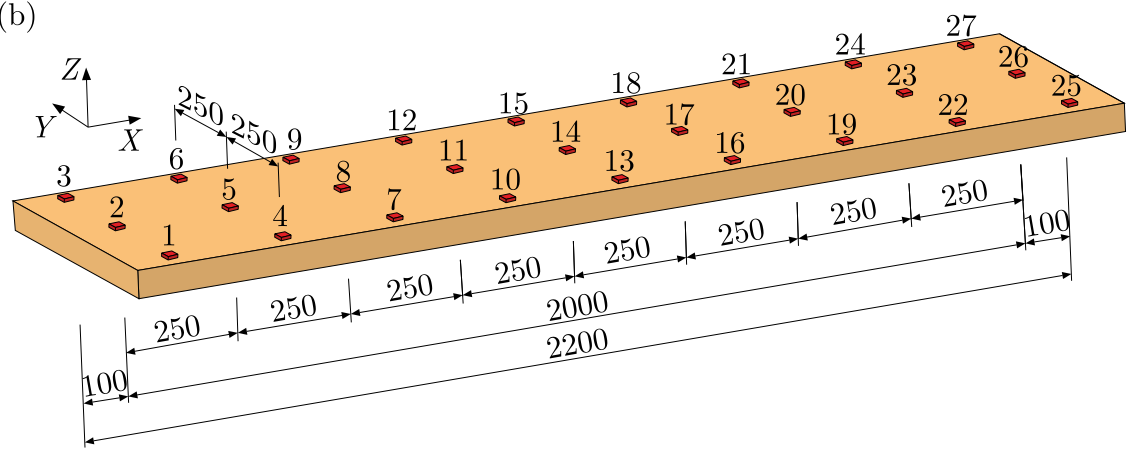

Fig. 2. Investigated slab: (a) excitation points, (b) measurement point grid

In Fig. 3, some mode shapes of the concrete slab obtained during the dynamic research are presented.

Dynamic characteristics of the frequency response functions were obtained and used to identify the coefficient of damping and other parameters. Peak amplitude values of FRF runs were determined. The peak amplitude values were corresponding to the next resonance vibrations of the reinforced concrete slab. Two types of vibrations were analysed: flexural vibrations (impulse excitation $2-Z$ ) and torsional vibrations (impulse excitation $1-Z$ ). For flexural and torsional vibrations, the peak amplitude values were determined for five resonances.

The authors developed their own software in MATLAB, which automatically defines the peak amplitude of FRF runs corresponding to the resonance vibration of the reinforced concrete slab. The software enabled determination of the values in tabular and graphical forms. For the analysis of FRF runs, eight representative measurement points were selected. For flexual vibration (impulse excitation $2-Z$ ) points 2, 5, 23 and 26 were selected, which were located on the axis of the reinforced concrete slab (see Fig. 2b). For torsional vibration (impulse excitation $1-Z$ ) points 1, 3, 25 and 27 were selected, which were located on the corners of the reinforced concrete slab (see Fig. 2b). Peak amplitudes of the run of FRF corresponding resonances are summarised in Table 2. 
(a)

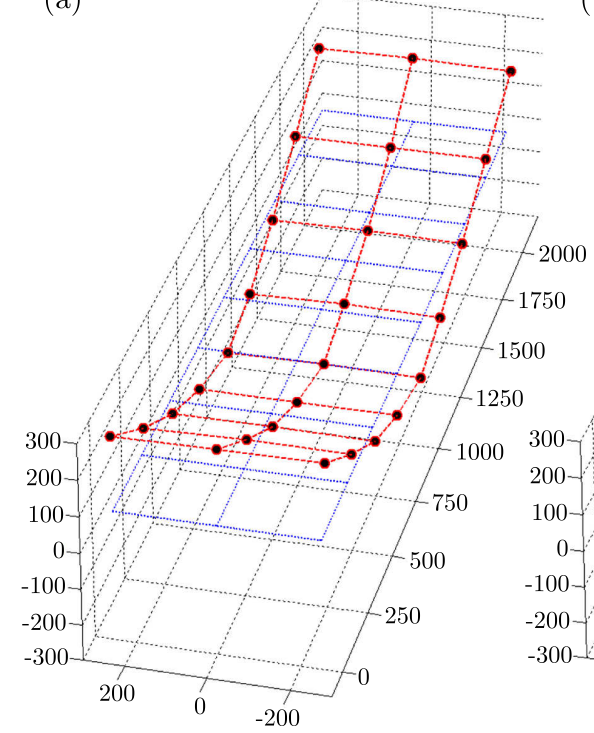

(b)

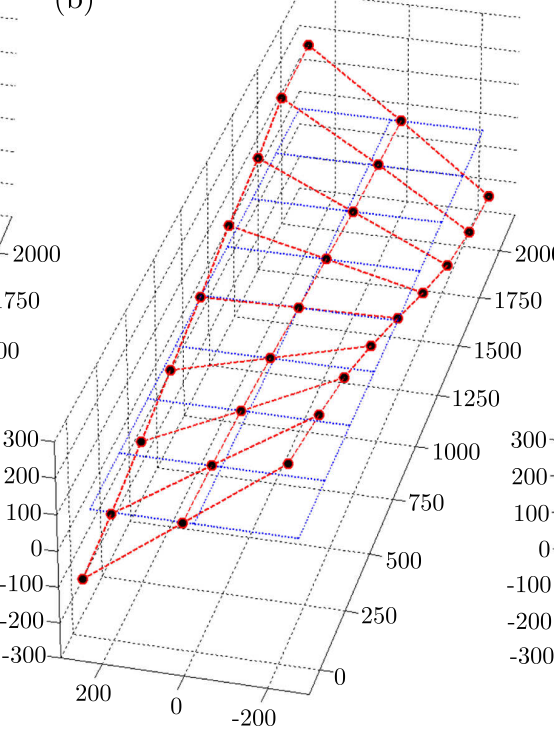

(c)

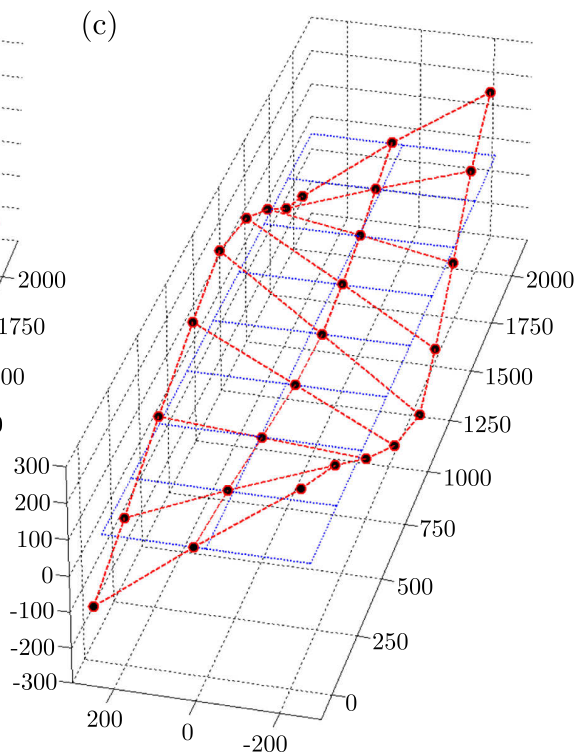

Fig. 3. Flexural and torsional experimental mode shapes for the reinforced concrete slab, excitation $1-Z$ : (a) $1_{\text {flex }}=50.04 \mathrm{~Hz}$, (b) $1_{\text {tors }}=113.34 \mathrm{~Hz}$, (c) $2_{\text {tors }}=232.75 \mathrm{~Hz}$

Table 2. Amplitude of frequency response functions for the reinforced concrete slab determined during measurement, impulse excitation $1-Z$, direction $Z$

\begin{tabular}{|c|c|c|c|c|c|c|c|c|}
\hline Points & \multicolumn{2}{|c|}{1} & \multicolumn{2}{c|}{3} & \multicolumn{2}{c|}{25} & \multicolumn{2}{|c|}{27} \\
\hline FRF & $\begin{array}{c}\mathrm{FRF} \\
{\left[\mathrm{m} \cdot \mathrm{s}^{-2} / \mathrm{N}\right]}\end{array}$ & $\begin{array}{c}f_{i}^{\text {exp }} \\
{[\mathrm{Hz}]}\end{array}$ & $\begin{array}{c}\mathrm{FRF} \\
{\left[\mathrm{m} \cdot \mathrm{s}^{-2} / \mathrm{N}\right]}\end{array}$ & $\begin{array}{c}f_{i}^{\text {exp }} \\
{[\mathrm{Hz}]}\end{array}$ & $\begin{array}{c}\mathrm{FRF} \\
{\left[\mathrm{m} \cdot \mathrm{s}^{-2} / \mathrm{N}\right]}\end{array}$ & $\begin{array}{c}f_{i}^{\text {exp }} \\
{[\mathrm{Hz}]}\end{array}$ & $\begin{array}{c}\mathrm{FRF} \\
{\left[\mathrm{m} \cdot \mathrm{s}^{-2} / \mathrm{N}\right]}\end{array}$ & $\begin{array}{c}f_{i}^{\text {exp }} \\
{[\mathrm{Hz}]}\end{array}$ \\
\hline \hline $1_{\text {tors }}$ & 1.915 & 113 & 2.000 & 113 & 1.968 & 113 & 1.966 & 113 \\
\hline $2_{\text {tors }}$ & 3.117 & 233 & 3.162 & 233 & 3.152 & 233 & 3.165 & 233 \\
\hline $3_{\text {tors }}$ & 2.63 & 368 & 2.599 & 368 & 2.676 & 367 & 2.598 & 367 \\
\hline $4_{\text {tors }}$ & 2.077 & 526 & 2.051 & 526 & 2.022 & 526 & 1.992 & 526 \\
\hline $5_{\text {tors }}$ & 2.632 & 708 & 0.51 & 707 & 2.321 & 708 & 0.553 & 709 \\
\hline
\end{tabular}

In Fig. 4, frequency response functions with marked peak amplitude values (from torsional vibrations) for point 27 for direction $Z$, impulsive excitation $1-Z$ are presented.

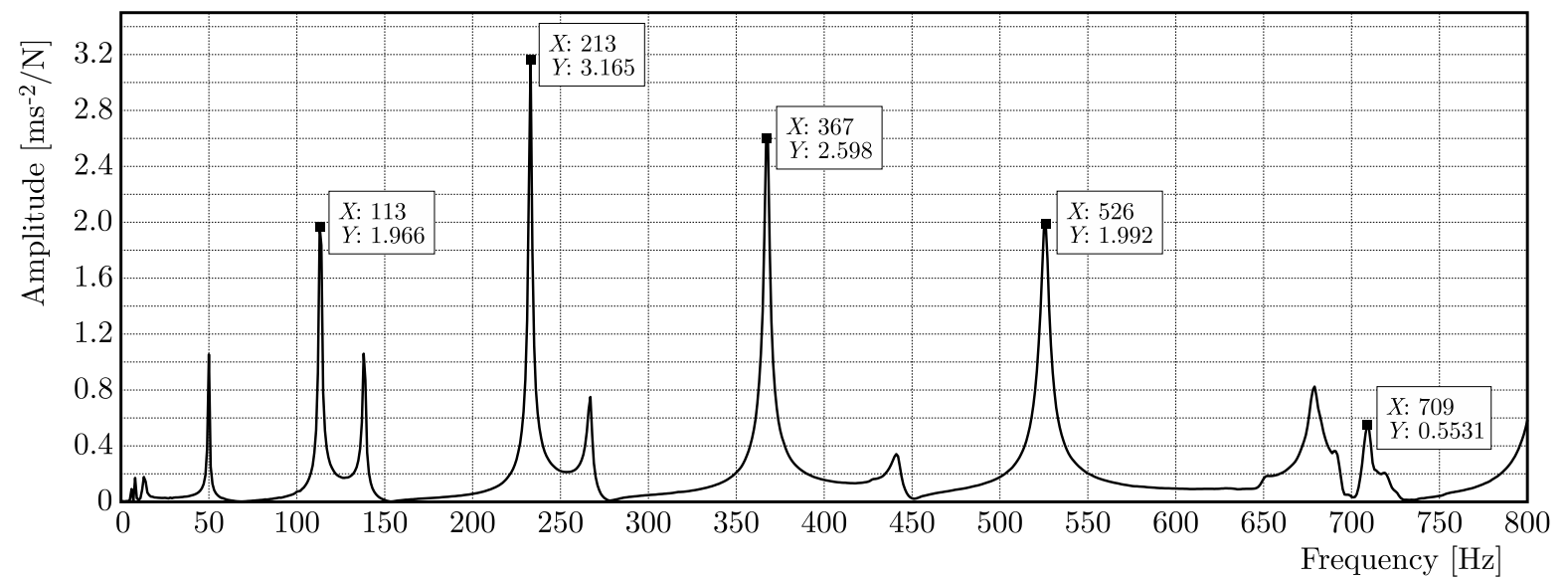

Fig. 4. Amplitude of the frequency response functions for the reinforced concrete slab, excitation $1-Z$, point 27 , direction $Z$ 


\section{The spatial computational model of the reinforced concrete slab}

A discrete computational model of the slab has been developed in the convention of the Rigid Finite Element Method. The method consists in dividing a real system into rigid finite elements which are represented by spring-damping elements (SDEs). While rigid finite elements (RFEs) are characterised by masses and mass moments of inertia, spring-damping elements are defined by stiffness and damping coefficients. The RFE method was developed by Kruszewski et al. (1999) and Wittbrodt et al. (2006).

Modelling of continuous elements in the finite element method starts from primary segmentation. For a slab, the segmentation must be conducted in two directions, i.e. in the longitudinal direction along $\Delta L$ and in transverse direction along $\Delta B$. The model is divided into segments of equal or comparable length. The primary segmentation of the present study is shown in Fig. 5a. Then, at the center of gravity of each element, an SDE is placed which focuses spring and damping properties of that element. Each SDE is broken down into four smaller SDEs, so that it is possible to connect the corners of four adjacent finite elements - this is secondary segmentation. In every set of four SDEs, two of them are parallel to the main axis $X$ and the other two are parallel to the main axis $Y$. In the classic approach (Kruszewski et al., 1999) spring properties of respective elements are reflected by SDEs spaced as shown in Fig. 5b.


Fig. 5. 3D finite element method: (a) primary segmentation, (b) secondary segmentation - classic positioning of SDEs, (c) secondary segmentation - modified positioning of SDEs

In the classic approach, the elements have 5 degrees of freedom. By placing an SDE at the corners, it is possible to neglect rotation in the axes perpendicular to the area of a primary element. The proposed model attempts to define the slab with 6 degrees of freedom (three translational and three rotational displacements). In order to limit element rotation in the axis 
perpendicular to the area of the primary element, SDEs must be moved from the corners to the centers of finite elements as shown in Fig. 5c. A similar approach was used in (Adamiec-Wójcik and Wojciech, 2012).

Each rigid finite element has its own independent coordinate system $X_{R F E}^{(i)}, Y_{R F E}^{(i)}, Z_{R F E}^{(i)}$ which is selected so that it overlaps the principal central axes of inertia of a given RFE. Given this assumption, mass and moments of inertia are the only parameters necessary to describe any $\mathrm{RFE}$. These quantities can be given in form of a diagonal mass matrix

$$
\mathbf{M}^{(i)}=\operatorname{diag}\left[m^{(i)}, m^{(i)}, m^{(i)}, J_{X}^{(i)}, J_{Y}^{(i)}, J_{Z}^{(i)}\right]
$$

The first three terms of the matrix are equal to the mass of the RFE, while the other three are RFE mass moments of inertia relative to the axes $X_{R F E}^{(i)}, Y_{R F E}^{(i)}, Z_{R F E}^{(i)}$. The values of the diagonal element of the mass matrix $\mathbf{M}^{(i)}$ for modelling of RFEs inside the slab are determined in the following way

$$
m^{(i)}=h_{c} \Delta L \Delta B \rho_{c}
$$

where $h_{c}$ is thickness of the reinforced concrete slab, $\rho_{c}$ - mass density of RFE material

$$
\begin{array}{ll}
J_{X}^{(i)} & =\frac{m^{(i)}}{12}\left(\Delta L^{2}+h_{c}^{2}\right) \\
J_{Z}^{(i)} & =\frac{m^{(i)}}{12}\left(\Delta L^{2}+\Delta B^{2}\right)
\end{array}
$$

Every SDE of $k$ number has its own independent coordinate system with the main axes $X_{S D E}^{(k)}$, $Y_{S D E}^{(k)}, Z_{S D E}^{(k)}$. The main axes of the SDE have such property that forces acting on the SDE in a direction compatible with these axes result in its translational deformations which occur only in the direction along which these forces are applied. The main parameters which describe an SDE of $k$ number are coefficients defining its spring and damping properties. The spring properties are described by means of two matrices: a matrix of translational stiffness coefficients $\mathbf{K}_{T}^{(k)}$ and a matrix of rotational stiffness coefficients $\mathbf{K}_{R}^{(k)}$. Both matrices are diagonal and they are $3 \times 3$ in size

$$
\mathbf{K}_{T}^{(k)}=\operatorname{diag}\left[k_{T, X}^{(k)}, k_{T, Y}^{(k)}, k_{T, Z}^{(k)}\right] \quad \mathbf{K}_{R}^{(k)}=\operatorname{diag}\left[k_{R, X}^{(k)}, k_{R, Y}^{(k)}, k_{R, Z}^{(k)}\right]
$$

The values of translational and rotational stiffness coefficients are determined according to the following rules:

— for SDEs parallel to the main axis $X$

$$
\begin{array}{ll}
k_{T, X-X}^{(k)}=\frac{E_{c} h_{c, \text { eff }} \Delta B}{\Delta L} & k_{T, Y-X}^{(k)}=\frac{G_{c} h_{c, e f f} \Delta B}{\Delta L \chi} \\
k_{T, Z-X}^{(k)}=\frac{G_{c} h_{c, e f f} \Delta B}{\Delta L \chi} & k_{R, X-X}^{(k)}=\frac{G_{c} h_{c, e f f}^{3} \Delta B}{6 \Delta L} \\
k_{R, Y-X}^{(k)}=\frac{E_{c} h_{c, e f f}^{3} \Delta B}{12\left(1-\nu_{c}^{2}\right) \Delta L} & k_{T, Z-X}^{(k)}=\frac{E_{c} h_{c, e f f} \Delta B^{3}}{12 \Delta L}
\end{array}
$$

- for SDEs parallel to the main axis $Y$

$$
\begin{aligned}
k_{T, X-Y}^{(k)}=\frac{G_{c} h_{c, e f f} \Delta L}{\Delta B \chi} & k_{T, Y-Y}^{(k)}=\frac{E_{c} h_{c, e f f} \Delta L}{\Delta B} \\
k_{T, Z-Y}^{(k)}=\frac{G_{c} h_{c, e f f} \Delta L}{\Delta B \chi} & k_{R, X-Y}^{(k)}=\frac{E_{c} h_{c, e f f}^{3} \Delta L}{12\left(1-\nu_{c}^{2}\right) \Delta B} \\
k_{R, Y-Y}^{(k)}=\frac{G_{c} h_{c, e f f}^{3} \Delta L}{6 \Delta B} & k_{T, Z-Y}^{(k)}=\frac{E_{c} h_{c, e f f} \Delta L^{3}}{12 \Delta B}
\end{aligned}
$$


where $E_{c}$ is the substitute dynamic longitudinal modulus of elasticity of the reinforced concrete slab (which takes into account the effect of reinforcement used), $G_{c}$ - substitute dynamic transverse modulus of elasticity of the reinforced concrete slab (which takes into account the effect of reinforcement used), $h_{c, e f f}$ - effective thickness of the reinforced concrete slab, $\nu_{c}$ - Poisson's ratio of concrete, $\chi$ - coefficient of cross-section shape (Timoshenko shear coefficient), which takes into account the nonuniform tangential stress pattern. The method of calculating the coefficient was described by Berczyński and Wróblewski (2010). The Timoshenko shear coefficient of cross-section of a rectangle shape is 1.2.

Dumping properties are described by means of two matrices of damping coefficients: $\mathbf{C}_{T}^{(k)}$ and $\mathbf{C}_{R}^{(k)}$. Both matrices are diagonal and they are $3 \times 3$ in size. The relation between equivalent stiffness $k_{i, j}^{(k)}$ and damping coefficients $c_{i, j}^{(k)}$ can be given by

$$
c_{i, j}^{(k)}=\frac{\eta}{\omega} k_{i, j}^{(k)} \quad i=T, R \quad j=X, Y, Z
$$

where $\eta$ is the loss ratio, $\omega$ - vibration frequency.

Results obtained with the modified RFE method model were compared with the analytical solution, the TM (Theoretical Model), and with the FEM model with flexible and deformable rigid bodies implemented in the Finite Element Method. The FEM model was developed in Abaqus environment and it was used for calculations. The slab was modelled with $50 \times 50$ second-order cubic elements with reduced integration for second-order functions (C3D20R). The solution was developed and reported by Liew et al. (1993), Leissa (1973). Leiss (1973) provided a solution for thin slabs, whereas Liew et al. (1993) found a solution of eigenmodes for medium-thick rectangular slabs. The authors presented their findings for various boundary conditions (21 cases) for different side length/width ratios $(a / b)$ and different slab thickness/width ratios $(h / b)$. Their approach was based on the energy function defined using the Mindlin theory of plates with the Rayleigh-Ritz minimisation procedure, providing a solution to the eigenvalue problem (Szcześniak, 2000). According to the theory of plates of medium thickness, it is typically assumed that a plate has thickness greater or equal $1 / 10$ of the smaller of the remaining two dimensions. The reinforced concrete slab used in our tests had the thickness to width ratio of $60 / 600$, i.e. $1 / 10$ precisely. Therefore, the theory of medium-thick plates could be applied for the modelling of both the former and the latter. The results were compared for a free-ends slab (the same scheme was used in empirical investigations). Liew et al. (1993) conducted analytical calculations for a slab with various length/width ratios for comparison purposes. Here, a $1500 \mathrm{~mm}$ long, $600 \mathrm{~mm}$ wide and $60 \mathrm{~mm}$ thick slab was used in the tests. Therefore, the length to width ratio of 2.5 and the thickness to width ratio of 0.1 was obtained. The Poisson ratio $\mu_{c}$ was 0.3 , Young's modulus $E_{c}$ was $3.0 \cdot 10^{10} \mathrm{~N} / \mathrm{m}^{2}$ and the mass density $\rho_{c}$ was $2400 \mathrm{~kg} / \mathrm{m}^{3}$. In an earlier work (Liew et al., 1993), the results were presented in a dimensionless form

$$
\lambda=\frac{\omega B^{2}}{\pi^{2}} \sqrt{\frac{\rho_{c} h_{c}}{D}}
$$

where $\omega$ is the angular frequency, $B$ - length of the shorter side of the slab width, $D$ - bending flexural stiffness of the slab.

$$
D=\frac{E_{c} h_{c}^{3}}{12\left(1-\nu_{c}^{2}\right)}
$$

The results are presented in Table 3. The calculations for the RFE model were conducted for the initial mesh of $30 \times 12$ elements, the slab dimensions were the same as those given above. Table 3 presents a comparison of solutions for the three models: the theoretical model (TM) based on the precise solution, the Rigid Finite Element (RFE) model and the Finite Element Method (FEM) model. 
Table 3. A comparison of dimensionless vibration frequencies of the slab for the TM, RFE and FEM models

\begin{tabular}{|c|c|c|c|c|c|c|}
\hline \multicolumn{7}{|c|}{ Reinforced concrete slab $60 \mathrm{~mm} \times 600 \mathrm{~mm} \times 1500 \mathrm{~mm}$} \\
\hline & \multirow{2}{*}{ Vibration forms of the slab } & TM & \multicolumn{2}{|c|}{ RFE } & \multicolumn{2}{|c|}{ FEM } \\
\hline 1 & & $\lambda_{i-T M}$ & $\lambda_{i_{-} R F E}$ & $\Delta_{i-T M}$ & $\lambda_{i_{-} F E M}$ & $\Delta_{i-T M}$ \\
\hline 1 & & 0.3455 & 0.3605 & $4.3 \%$ & 0.3457 & $0 \%$ \\
\hline 2 & & 0.5137 & 0.5144 & $0.1 \%$ & 0.5154 & $0 \%$ \\
\hline 3 & & 0.9486 & 0.9826 & $3.6 \%$ & 0.9502 & $0 \%$ \\
\hline 4 & & 1.0952 & 1.0939 & $-0.1 \%$ & 1.0994 & $0 \%$ \\
\hline 5 & & 1.8109 & 1.8080 & $-0.2 \%$ & 1.8199 & $0 \%$ \\
\hline 6 & & 1.8220 & 1.8952 & $4.0 \%$ & 1.8282 & $0 \%$ \\
\hline 7 & & 2.1919 & 2.1860 & $-0.3 \%$ & 2.1981 & $0 \%$ \\
\hline 8 & & 2.3566 & 2.3884 & $1.4 \%$ & 2.3654 & $0 \%$ \\
\hline 9 & & 2.7173 & 2.7164 & $0 \%$ & 2.7332 & $0.6 \%$ \\
\hline
\end{tabular}

The terms $\Delta_{i_{-} R F E}$ and $\Delta_{i_{-} F E M}$ defined by (4.10) are used to show the percentage differences for dimensionless values of slab frequency vibrations obtained for the TM, RFEM and FEM models.

$$
\Delta_{i_{-} R F E}=\frac{\lambda_{i_{-} R F E}-\lambda_{i_{-} T M}}{\lambda_{i_{-} T M}} \quad \Delta_{i_{-} F E M}=\frac{\lambda_{i_{-} F E M}-\lambda_{i_{-} T M}}{\lambda_{i_{-} T M}}
$$

The first column shows the obtained vibration forms of the slab for the dimensionless values of the slab frequencies.

A very good convergence of the results was obtained for the RFE and TM models. The largest difference was $4.3 \%$. The largest discrepancy between the FEM and TM models was observed for higher eigenmodes ( $0.6 \%$ for 9 th eigenmode). However, the results obtained for the torsional (2, 4, 5 and 9) and flexural vibrations (1,3 and 6), which are significant for parameter estimation of composite beams, were quite convergent for both the former and the latter.

During the analysis of various values of the parameter $\Delta$ for various mesh densities in the $X$ axis, a constant mesh density of 12 was used for the $Y$ axis. In the second case, with various mesh densities in the $Y$ axis, a constant mesh density of 30 was used for the $X$ axis. It was 
examined that for other constants for $X$ and $Y$ axes the same results were obtained. It was found that with an increase in mesh density, the eigenmode frequencies were asymptotically approaching the solution for the sufficient mesh density which provided a good convergence with the analytical solution. Nevertheless, the problem of mesh density selection was also affected by the fact that larger densities significantly increased calculation time.

\section{Parameter estimation - algorithm I}

The estimated parameters included the substitute longitudinal modulus of elasticity of the reinforced concrete slab $E_{c}$ which took into account longitudinal reinforcement and effective thickness of the reinforced concrete slab $h_{c, e f f}$. Other parameters used for the identification of the computational model were taken from the literature or from the design of the analysed slab.

The differential equation of free vibration obtained from the general differential equation of motion, while neglecting outside interaction acting on the slab and damping effects, can be presented as

$$
\mathbf{M} \ddot{\mathbf{q}}+\mathbf{K q}=\mathbf{0}
$$

where $\mathbf{q}$ is the vector of generalised coordinates.

Methods of solving equation (5.1) to determine free vibration frequencies and corresponding vibration modes are described in detail in literature (Wilde, 2008).

The quantities $S_{\text {flex }}$ and $S_{\text {tors }}(5.2)$ are sums of squares of the relative deviations of the first $n$ numerical frequencies of flexural and torsional vibrations with their analogous frequencies determined during the experiment

$$
S_{\text {flex }}=\sum_{i=1}^{n}\left(\frac{f_{i_{-} \text {flex }}^{\text {num }}-f_{i_{-} \text {flex }}^{\text {exp }}}{f_{i_{-} \text {flex }}^{\text {exp }}}\right)^{2} \quad S_{\text {tors }}=\sum_{i=1}^{n}\left(\frac{f_{i_{-} \text {tor }}^{\text {num }}-f_{i_{-} \text {tors }}^{\text {exp }}}{f_{i_{-} \text {tors }}^{\text {exp }}}\right)^{2}
$$

where $n$ is the number of selected frequencies, $f_{i_{-} \text {flex }}^{\text {exp }}$ - experimental/numerical frequencies of flexural vibrations, $f_{i \_t o r s}^{\text {exp }}$ - experimental/numerical frequencies of torsional vibrations.

The quantity $S$ is a sum of flexural and torsional vibrations in the process of estimation in which an appropriate weight function $w_{\text {flex }}$ was attributed to flexural and wtors to torsional frequencies

$$
S=w_{\text {flex }} S_{\text {flex }}+w_{\text {tors }} S_{\text {tors }}
$$

An additional criterion imposed was the condition of compatibility of the experimental and mathematical fundamental frequency of longitudinal vibrations:

$$
f_{1 \_l o n g}^{\text {exp }}=f_{1 \_ \text {long }}^{\text {num }}
$$

The algorithm assumes a limit (5.4) that has the greatest impact on the identified slab modulus of elasticity $E_{c}$.

In order to determine model parameters that allow the best mapping of frequencies with those obtained experimentally, the paramter $S$ index was minimised. To solve the problem, an optimisation procedure implemented in Optimization Toolbox package, which is a part of MATLAB, was used.

For adopted criterion (5.3), the following numerical experiments was performed. In the 1st stage of parameter estimation criterion (5.3) was minimised assuming $w_{\text {flex }}=1.0$ and $w_{\text {tors }}=0.0$. This means comparison of only flexural frequencies of natural vibration during the estimation 
process. The estimation resulted in a good compatibility of frequencies of natural vibration observed in the experiments and in numerical calculations (see Table 4 - Analysis I). Differences in compatibility for flexure frequencies for the reinforced concrete slab were under $1.4 \%$ and for torsional frequencies under $3.1 \%$. The iteration algorithm was tested for convergence by introducing various starting points to determine parameters of the the model. Each time, after the iteration was complete, the values of estimated variables were similar.

Table 4. Comparison of natural vibration frequencies measured during dynamic tests with numerical results algorithm I of the estimation, Analysis I-III

\begin{tabular}{|c|c|c|c|c|c|c|c|c|c|}
\hline Analysis & \multicolumn{3}{|c|}{$\mathrm{I}$} & \multicolumn{3}{|c|}{ II } & \multicolumn{3}{|c|}{ III } \\
\hline FRF & $\begin{array}{l}f_{i}^{e x p} \\
{[\mathrm{~Hz}]}\end{array}$ & $\begin{array}{l}f_{i}^{\text {num }} \\
{[\mathrm{Hz}]}\end{array}$ & $\begin{array}{c}\Delta \\
{[\%]}\end{array}$ & $\begin{array}{l}f_{i}^{e x p} \\
{[\mathrm{~Hz}]}\end{array}$ & $\begin{array}{l}f_{i}^{\text {num }} \\
{[\mathrm{Hz}]}\end{array}$ & $\begin{array}{c}\Delta \\
{[\%]}\end{array}$ & $\begin{array}{l}f_{i}^{\exp } \\
{[\mathrm{Hz}]}\end{array}$ & $\begin{array}{l}f_{i}^{\text {num }} \\
{[\mathrm{Hz}]}\end{array}$ & $\begin{array}{c}\Delta \\
{[\%]}\end{array}$ \\
\hline $1_{\text {tors }}$ & 113.34 & 110.2 & -2.8 & 113.34 & 113.4 & 0.1 & 113.34 & 111.33 & -1.8 \\
\hline $22_{\text {tors }}$ & 232.75 & 227.4 & -2.3 & 232.75 & 233.97 & 0.5 & 232.75 & 229.7 & 1.3 \\
\hline 3 & 367.33 & 358.33 & -2.5 & 367.33 & 368.61 & 0.3 & 367.33 & 361.94 & -1.5 \\
\hline $4_{\text {tors }}$ & 525.61 & 509.55 & -3.1 & 525.61 & 524.03 & -0.3 & 525.61 & 514.64 & -2.1 \\
\hline 5 tors & 707.99 & 686.87 & -3.0 & 707.99 & 706.18 & -0.3 & 707.99 & 693.65 & -2.0 \\
\hline $1_{\text {flex }}$ & 50.00 & 50.64 & 1.3 & 50.00 & 52.13 & 4.3 & 50.00 & 51.16 & 2.3 \\
\hline 2 flex & 138.34 & 138.94 & 0.4 & 138.34 & 143.00 & 3.4 & 138.34 & 140.36 & 1.5 \\
\hline $3_{\text {flex }}$ & 266.76 & 270.53 & 1.4 & 266.76 & 278.36 & 4.3 & 266.76 & 273.28 & 2.4 \\
\hline $4_{\text {flex }}$ & 441.54 & 443.22 & 0.4 & 441.54 & 455.9 & 3.3 & 441.54 & 447.67 & 1.4 \\
\hline $5_{\text {flex }}$ & 649.34 & 654.85 & 0.8 & 649.34 & 673.31 & 3.7 & 649.34 & 661.34 & 1.8 \\
\hline $1_{\text {long }}$ & 933.12 & 933.12 & 0.0 & 933.12 & 933.12 & 0.0 & 933.12 & 933.12 & 0.0 \\
\hline$E_{c}\left[\mathrm{~N} / \mathrm{m}^{2}\right]$ & \multicolumn{3}{|c|}{$4.248 \mathrm{E}+10$} & \multicolumn{3}{|c|}{$4.127 \mathrm{E}+10$} & \multicolumn{3}{|c|}{$4.205 \mathrm{E}+10$} \\
\hline$h_{c, f f}[\mathrm{~m}]$ & \multicolumn{3}{|c|}{0.057} & \multicolumn{3}{|c|}{0.059} & \multicolumn{3}{|c|}{0.058} \\
\hline$S_{\text {tors }}$ & \multicolumn{3}{|c|}{$3.718 \mathrm{E}-03$} & \multicolumn{3}{|c|}{$5.500 \mathrm{E}-05$} & \multicolumn{3}{|c|}{$1.548 \mathrm{E}-03$} \\
\hline$w_{\text {tors }}$ & \multicolumn{3}{|c|}{0.0} & \multicolumn{3}{|c|}{1.0} & \multicolumn{3}{|c|}{1.0} \\
\hline$S_{\text {flex }}$ & \multicolumn{3}{|c|}{$4.711 \mathrm{E}-04$} & \multicolumn{3}{|c|}{$7.262 \mathrm{E}-03$} & \multicolumn{3}{|c|}{$1.890 \mathrm{E}-03$} \\
\hline$w_{\text {flex }}$ & \multicolumn{3}{|c|}{1.0} & \multicolumn{3}{|c|}{0.0} & \multicolumn{3}{|c|}{1.0} \\
\hline$S$ & \multicolumn{3}{|c|}{$4.711 \mathrm{E}-04$} & \multicolumn{3}{|c|}{$5.500 \mathrm{E}-05$} & \multicolumn{3}{|c|}{$3.438 \mathrm{E}-03$} \\
\hline
\end{tabular}

The following Section presents results of the 2nd stage of estimation for weight functions $w_{\text {flex }}=0.0$ and $w_{\text {tors }}=1.0$. This means comparison of only torsional frequencies of natural vibration during the estimation process. The estimation results are presented in Table 4 - Analysis II. Differences in compatibility for torsional frequencies for the reinforced concrete slab were under $0.5 \%$ and for flexural frequencies under $4.3 \%$.

The following part of the paper presents results of the 3rd stage of estimation for weight functions $w_{f l e x}=1.0$ and $w_{\text {tors }}=1.0$. This means comparison of flexual and torsional frequencies of natural vibration during the estimation process. The estimation results are presented in Table 4 - Analysis III. Differences in compatibility for torsional frequencies for the reinforced concrete slab were under $2.1 \%$ and for flexural frequencies under $2.4 \%$.

\section{Parameter estimation for the model - algorithm II}

While working on the finite element method model of the slab, an assumption was made that the substitute dynamic longitudinal modulus of elasticity of the reinforced concrete slab $E_{c}$, effective thickness of the reinforced concrete slab $h_{c, \text { eff }}$, and the loss ratio of concrete $\eta_{c}$ would be determined based on identification. The value of the loss ratio is dependent on frequency, temperature and other factors. 
The loss ratio of concrete varies in the range (2-6) $10^{-4}$ according to (Silva, 2000). High diversity of concrete types results in a very different range of concrete damping values. Damping depends on concrete density, amount of cement slurry, load history, intensity of stress, etc.

Parameter estimation was conducted by fitting the frequency response functions calculated with the finite element method model to the characteristics obtained in experimental research. A system of differential equations defining the oscillating motion with damping can be given by

$$
\mathbf{M} \ddot{\mathbf{q}}(t)+\mathbf{C} \dot{\mathbf{q}}(t)+\mathbf{K q}(t)=\mathbf{f}(t)
$$

where $\mathbf{q}$ is the vector of generalised displacement, $\mathbf{M}, \mathbf{C}, \mathbf{K}$ - inertia, dumping and stiffness matrices, $\mathbf{f}$ - vector of generalised forces. The vectors $\mathbf{q}$ and the system response $\mathbf{f}$ are functions of time $t$. The above system of differential equations can be solved, depending on the form of the excitation signal, using either Fourier or Laplace integral transform (Marchelek, 1991). By using the Laplace transform, it is possible to move from time domain over to the domain of the complex frequency $s$. Given zero initial conditions, while performing the Laplace transform, the system of equations (6.1) takes the following form

$$
\left(\mathbf{M} s^{2}+\mathbf{C} s+\mathbf{K}\right) \mathbf{q}(s)=\mathbf{f}(s)
$$

A consequence of using the Laplace transform is algebraisation of the system of equations (6.1). While solving a system of linear algebraic equations, we assume that the matrix $\left(\mathbf{M} s^{2}+\mathbf{C} s+\mathbf{K}\right)$ is not singular, i.e. that there is a matrix inverse to it. As a result, Equation (6.2) takes the form

$$
\mathbf{q}(s)=\left(\mathbf{M} s^{2}+\mathbf{C} s+\mathbf{K}\right)^{-1} \mathbf{f}(s)
$$

To find a solution in the frequency form, if the excitation applied to the system is periodic (solution for a steady state), the Fourier transform can be used. A solution is found directly in the Laplace solution by substituting it to Equation (6.3) $s=\mathrm{j} \omega$, where $\mathrm{j}=\sqrt{-1}$

$$
\mathbf{q}(\mathrm{j} \omega)=\left(\mathbf{K}-\omega^{2} \mathbf{M}+\mathrm{j} \omega \mathbf{C}\right)^{-1} \mathbf{f}(\mathbf{j} \omega)
$$

where

$$
\mathbf{A}(\mathrm{j} \omega)=\mathbf{K}-\omega^{2} \mathbf{M}+\mathrm{j} \omega \mathbf{C}
$$

is referred to as the dynamic stiffness matrix, while

$$
\mathbf{W}(\mathrm{j} \omega)=\mathbf{A}^{-1}(\mathrm{j} \omega)=\left(\mathbf{K}-\omega^{2} \mathbf{M}+\mathrm{j} \omega \mathbf{C}\right)^{-1}
$$

is the dynamic flexibility matrix.

Dynamic flexibility is a characteristic obtained on the premise that the system input is a force and its output is a displacement. During experimental research, acceleration was measured. A characteristic found given the condition that the system input is the force and its output is the displacement is called inertance $\mathbf{G}(\mathrm{j} \omega)$. Both dynamic flexibility and inertance are frequency characteristics defined for steady motion and they are therefore closely interrelated (Uhl, 1997).

$$
|\mathbf{G}(\mathbf{j} \omega)|=\omega^{2}|\mathbf{W}(\mathbf{j} \omega)|
$$

To find the inertance of a system based on a finite element model, it is necessary to know the stiffness matrix $\mathbf{K}$, inertia matrix $\mathbf{M}$ and damping matrix $\mathbf{C}$. The methods were described in-depth elsewhere in the literature (Kruszewski et al., 1999; Wróblewski et al., 2013).

Our identification criterion was minimization of the coefficient JFRF (6.7) which is a double sum for $m$-th measurement points, a sum of relative quadratic deviation of the first $n$-th measurement points for a given FRF amplitude to the same amplitude determined in experimental 
research. While determining loss ratios, an attempt was made to fit the calculated amplitudes with those determined experimentally

$$
J_{F R F}=\sum_{i=1}^{m} \sum_{i=1}^{n}\left(\frac{F R F_{i, a m p}^{\text {num }}-F R F_{i, a m p}^{\exp }}{F R F_{i, a m p}^{\exp }}\right)^{2}
$$

The above algorithm allowed one to select an analysed point and, as a result, it was possible to choose vibration forms (flexural and torsional vibration) and vibration modes $(1,2, \ldots, n)$. Both flexural and torsional vibration forms were taken into account in the estimation procedure. An optimization procedure implemented in Optimization Toolbox package, which is a part of MATLAB, was used.

The results of analysis are presented in Table 5. First analysis was conducted for torsional vibration for point 1 (vibration modes from 2 to 4 ) and for flexural vibration for point 2 (vibration modes from 2 to 4 ). In the identification, 5 points from each amplitude of every FRF were taken into consideration.

Table 5. Results of estimated parameters during optimisation - algorithm II of the estimation, Analysis I-III

\begin{tabular}{|l|c|c|c|}
\hline Analysis & I & II & III \\
\hline \hline$E_{c}\left[\mathrm{n} / \mathrm{m}^{2}\right]$ & $4.230 \mathrm{E}+10$ & $4.300 \mathrm{E}+10$ & $4.224 \mathrm{E}+10$ \\
\hline$h_{c, e f f}[\mathrm{~m}]$ & 0.0570 & 0.0577 & 0.0570 \\
\hline$\eta_{c}[-]$ & 0.0090 & 0.0112 & 0.0091 \\
\hline$J$ & 9.4703 & 4.7114 & 3.7924 \\
\hline
\end{tabular}

Second analysis was conducted for torsional vibration for point 1 (vibration modes from 2 to 4 ) and for flexural vibration for point 2 (vibration mode 3 ). In the identification, 5 points from each amplitude of every FRF were taken into consideration. Third analysis was performed for torsional vibration for point 1 (vibration mode 2 ) and for flexural vibration for point 2 (vibration modes from 2 to 4 ). In the identification, 5 points from each amplitude of every FRF were taken into consideration.


Measurment point 1-direction $Z$-excitation 1- $Z$

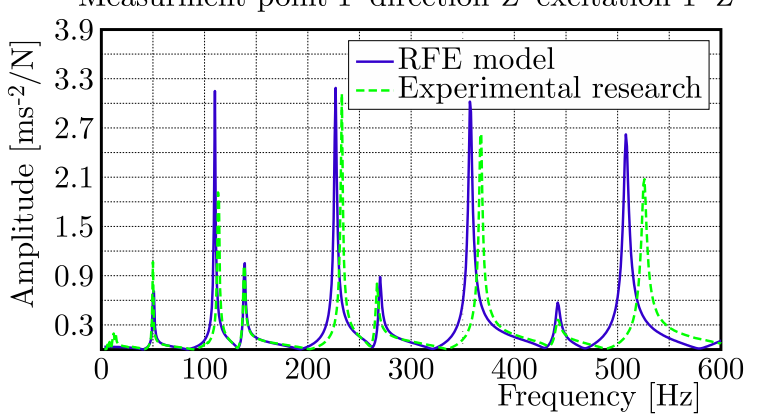

Measurment point 27-direction $Z$-excitation 1- $Z$

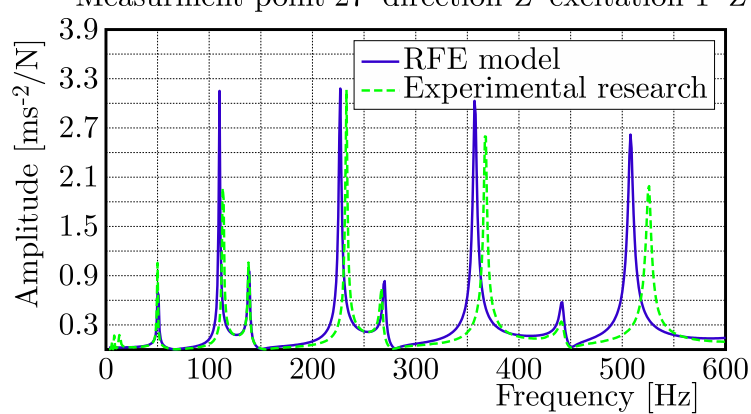

Fig. 6. A comparison of the frequency response functions for the reinforced concrete slab - Analysis III 
A comparison of FRFs determined using the above estimated parameters with those determined experimentally is presented in Fig. 6. As the consistency of the FRFs was high, it was fair to state that the identified parameters were determined correctly.

\section{Conclusions}

The numerical tests show a very good convergence of the algorithms developed for estimating the parameters of a mathematical model of the reinforced concrete slab. They enable creation of a model representing the actual reinforced concrete slab and allowed one to determine dynamic characteristics very similar to those measured during the experiments.

Following the identification, it can be observed that the identified parameters for algorithms I and II are very similar. Both algorithms provide convergent results of identification, which indicates the appropriateness of the model and algorithms.

A very good fit of FRFs calculated using the model with those obtained in the experimental research is achieved. The originally developed spatial finite element model allows one to take into account both flexural and torsional vibration forms. The goal was to achieve consistency of frequencies and FRFs observed during tests with those calculated using the analytical model.

This well-developed 3D model of the reinforced concrete slab has allowed the authors to develop a model of a steel-concrete composite beam which has been the focus of their research attempts.

\section{Acknowledgments}

The research work reported in this paper was made possible throught financial support from MNiSW Poland in the years 2011-2013, completed within the project N N501 192138.

\section{References}

1. AdAmiec-Wójcik I., Wojciech S., 2012, Rigid finite element method in modelling of vibrations of electrostatic precipitators (in Polish), Modelowanie Inżynierskie, 43, 7-14

2. BerczyŃski S., Gutowski P., 2006, Identification of the dynamic models of machine tool supporting systems. Part I: An algorithm of the method, Journal of Vibration and Control, 12, 279-295

3. Berczyński S., WróBlewski T., 2005, Vibration of steel-concrete composite beams using the Timoshenko beam model, Journal of Vibration and Control, 11, 829-848

4. Berczyński S., WróBlewski T., 2010, Experimental verification of natural vibration models of steel-concrete composite beams, Journal of Vibration and Control, 16, 2057-2081

5. Ewins D.J., 2000, Modal Testing: Theory, Practice, and Application, Hertfordshire, Research Studies Press

6. He J., Fu Z.F., 2001, Modal Analysis, Butterworth-Heinemann

7. Kruszewski J., Sawiak S., Wittbrodt E., 1999, The Rigid Finite Element Method in Structural Dynamics (in Polish), WNT, Warszawa

8. Leissa A.W., 1973, The free vibration of rectangular plates, Journal of Sound and Vibration, 31 , 3, 257-293

9. Liew K. M., Xiang Y., Kitipornchai S., 1993, Transverse vibration of thick rectangular plates. I - Comprehensive sets of boundary conditions, Computer and Structures, 49, 1, 1-29

10. MarcheleK K., 1991, Dynamics of Machine Tools (in Polish), WNT, Warszawa

11. Silva C.W.D, 2000, Vibration. Fundamentals and Practice, CRC Press, Boca Raton, FL. 
12. Szcześniak W., 2000, Selected Aspects of the Dynamics of Plates (in Polish), Oficyna Wydawnicza Politechniki Warszawskiej, Warszawa

13. Uhu T., 1997, Computer-Aided Identification of Models of Mechanical Structures (in Polish), WNT, Warszawa

14. Wilde K., 2008, Modal Diagnostics of Civil Engineering Structures, Gdańsk University of Technology

15. Wittbrodt E., Adamiec-Wójcik I., Wojciech S., 2006, Dynamics of Flexible Multibody Systems. Rigid Finite Element Method, Springer-Verlag, Berlin, Heidelberg, New York

16. Wróblewski T., Berczyński S., Abramowicz M., 2013, Estimation of the parameters of the discrete model of a steelconcrete composite beam, Archives of Civil and Mechanical Engineering, 13, 209-219

Manuscript received April 7, 2015; accepted for print August 23, 2016 